\title{
Oxigenoterapia Domiciliária de Longa Duração na Criança: Evidências e Questões em Aberto
}

\author{
Long-Term Home Oxygen Therapy in Children: Evidences and Open Issues
}

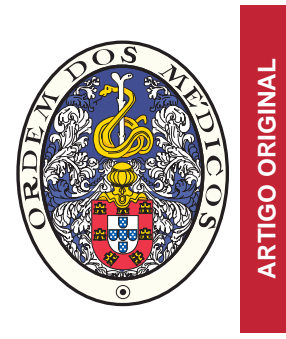

\author{
Lia OLIVEIRA ${ }^{1}$, Joana COELHO ${ }^{1}$, Rosário FERREIRA ${ }^{1,2}$, Teresa NUNES ${ }^{1,2}$, Ana SAIANDA ${ }^{1,2}$, Luísa PEREIRA ${ }^{1,2}$, \\ Teresa BANDEIRA ${ }^{1,2}$ \\ Acta Med Port 2014 Nov-Dec;27(6):717-725
}

\section{RESUMO}

Introdução: Oxigenoterapia domiciliária de longa duração está indicada em doentes com hipoxémia crónica. Pretendemos descrever a população em programa de oxigenoterapia domiciliária de longa duração acompanhada numa Unidade de Pneumologia Pediátrica de Hospital Terciário entre 2003-2012 e comparar com revisão de 1991-2000; verificar conformidade com orientações nacionais e internacionais, refletindo sobre necessidade de orientações nacionais especificamente pediátricas, inexistentes em Portugal.

Material e Métodos: Estudo retrospetivo, descritivo e comparativo por consulta de processo clínico. Pesquisa de orientações sobre oxigenoterapia em idade pediátrica.

Resultados: Incluímos 86 doentes (59,3\% rapazes). A idade mediana de início da oxigenoterapia foi 0,0 $(0,0-216,0)$ meses e a duração mediana de $15,0(3,0-223,0)$ meses. O diagnóstico mais frequente foi displasia broncopulmonar $(53,5 \%)$, seguindo-se bronquiolite obliterante $(14,0 \%)$, doença neurológica $(10,5 \%)$, fibrose quística $(8,1 \%)$, síndromes polimalformativas $(5,8 \%)$, doença de células falciformes $(3,5 \%)$, outras doenças pulmonares neonatais $(2,3 \%)$ e doenças pulmonares intersticiais $(2,3 \%)$. Mantêm acompanhamento $53(61,6 \%)$ doentes, 38 mantendo oxigenoterapia; $12(13,9 \%)$ faleceram. O tempo mediano de seguimento foi 39,5 (1,0-246,0) meses, mínimo nas outras doenças pulmonares neonatais e máximo na fibrose quística. Comparativamente ao estudo anterior revela aumento relativo dos lactentes com bronquiolite obliterante e displasia broncopulmonar, aumento da duração nestes últimos e inclusão de doentes neurológicos e hematológicos.

Discussão: A prescrição de oxigenoterapia domiciliária de longa duração em pediatria ocorre sobretudo em doenças específicas dos lactentes e idade pré-escolar. Doentes neurológicos e hematológicos são novos grupos de prescrição, à semelhança da literatura internacional.

Conclusão: O conhecimento da realidade nacional e orientações pediátricas são relevantes para organização de cuidados e prescrição racional.

Palavras-Chave: Criança; Cuidados de Longa Duração;Insuficiência Respiratória; Oxigenoterapia.

\section{ABSTRACT}

Introduction: Long-term home oxygen therapy is indicated for patients with chronic hypoxemia. We intend to describe pediatric population on long-term home oxygen therapy followed-up at Pediatric Respiratory Unit of a tertiary care hospital between 2003-2012 and to compare with previous 1991-2000 review; to verify conformity with international and national recommendations and need for specific pediatric national guidelines, non-existent in Portugal.

Material and Methods: Retrospective, descriptive and comparative study based on clinical files review. Review the guidelines for oxygen therapy in pediatric population.

Results: We studied 86 patients $(59.3 \%$ males). The median age at the beginning of oxygen therapy was $0.0(0.0-216.0)$ months, with a median duration of 15.0 (3.0-223.0) months. The most frequent diagnosis was bronchopulmonary dysplasia (53.5\%), followed by bronchiolitis obliterans $(14.0 \%)$, neurologic disorders $(10.5 \%)$, cystic fibrosis $(8.1 \%)$, miscellaneous syndromes $(5.8 \%)$, sickle-cell disease (3.5\%), other neonatal lung diseases (2.3\%) and interstitial lung diseases (2.3\%). Are maintained on follow-up $53(61.6 \%)$ patients, 38 on oxygen therapy; $12(13.9 \%)$ died. The median time of follow-up was $39.5(1.0-246.0)$ months, minim on other neonatal lung diseases and maximum on cystic fibrosis. Comparing with previous review, this shows a relative increase in bronchiolitis obliterans and bronchopulmonary dysplasia patients, with increased duration in the latter, and inclusion of neurologic and hematologic patients. Discussion: Prescription of long-term oxygen therapy in pediatric age mainly occurs in specific diseases of infants and pre-school aged. Neurologic and hematologic patients represent new indications, similarly to international publications.

Conclusion: The knowledge of national reality and pediatric orientations are needed for care plans and rational prescription.

Keywords: Child; Long-Term Care; Respiratory Insufficiency; Oxygen Inhalation Therapy; Portugal.

\section{INTRODUÇÃO}

O oxigénio terapêutico é utilizado em pediatria há mais de $60 \operatorname{anos}^{1}$ e de forma crescente na insuficiência respiratória crónica. ${ }^{2} \mathrm{~A}$ oxigenoterapia domiciliária de longa duração (OLD) tem especificidades pediátricas que motivaram orientações próprias ${ }^{3-8}$ na sequência de revisões casuísticas $^{2,5}$ ou de levantamentos nacionais de utilização. ${ }^{9} \mathrm{~A}$ utilização de oxigénio domiciliário favorece redução dos custos hospitalares, alta precoce e a redução da infeção associada aos cuidados de saúde..$^{10} \mathrm{~A}$ complexidade e especificidade da prescrição pediátrica de OLD, a amplitude de doenças candidatas e os custos associados, determinam a necessidade de coordenação de cuidados em centros de referência.

O aumento de OLD em idade pediátrica é consequência

1. Serviço de Pediatria Médica. Departamento de Pediatria. Hospital de Santa Maria. Centro Hospitalar Lisboa Norte. Lisboa. Portugal.

2. Unidade de Pneumologia Pediátrica. Cuidados Respiratórios Domiciliários e de Transição. Hospital de Santa Maria. Centro Hospitalar Lisboa Norte. Lisboa. Portugal. Recebido: 17 de Janeiro de 2014 - Aceite: 21 de Julho de 2014 | Copyright @ Ordem dos Médicos 2014 
da sobrevivência de grandes prematuros, alguns com displasia broncopulmonar, e de doentes complexos com doença pulmonar crónica. ${ }^{4,6}$

Sistemas de apoio a crianças dependentes de tecnologia permitem monitorizar parâmetros clínicos e funcionais e adequação terapêutica, evitando a deslocação do doente ao hospital e reduzindo o número de internamentos. A dinâmica com as famílias no domicílio permite prepará-las e auxilia-las na prestação de cuidados técnicos.

No doente adulto existem critérios de prescrição de OLD bem definidos, ${ }^{11}$ contudo na criança é mais complexo pela diversidade e complexidade das doenças. Em Portugal, ao contrário do que ocorre em Inglaterra, ${ }^{6}$ França $^{3}$ ou Brasil, ${ }^{2}$ faltam estudos que caracterizem a população pediátrica em OLD. Estes estudos permitem um melhor conhecimento da realidade, e favorecem a adequação dos cuidados e a elaboração de normas de orientação clínica especificamente pediátricas. Em Espanha existem também normas que fundamentam as indicações, métodos de administração, avaliações e seguimento. ${ }^{7} \mathrm{Em}$ Portugal, a Norma de Orientação Clínica (NOC) da Direção Geral de Saúde (DGS) para prescrição de oxigenoterapia $(018 / 2011)^{11}$ tece algumas considerações para a criança, mas não é específica para Pediatria.

No Reino Unido criou-se, em 2006, ${ }^{12}$ um sistema nacional de prescrição que permitiu conhecer e caracterizar a população pediátrica sob OLD, servindo de ponto de partida para a elaboração das normas de orientação clínica. ${ }^{7,8}$

Propusemo-nos, assim, caraterizar a população pediátrica submetida a OLD domiciliária acompanhada numa Unidade de Pneumologia Pediátrica de um Hospital Terciário num período de 10 anos (2003-2012) e comparar as características demográficas e doença de base com um estudo realizado na mesma Unidade na década 1991-2000. Foram objetivos secundários averiguar conformidade com orientações internacionais e nacionais e refletir sobre necessidade de orientações pediátricas.

\section{MATERIAL E MÉTODOS}

Estudo retrospetivo, descritivo e comparativo, efetuado por análise de processo clínico. Os doentes foram identificados através das bases de registos da Unidade de Pneumologia Pediátrica no período 2003-2012. Os dados foram consultados por observadores independentes do processo de acompanhamento clínico.

Foram critérios de inclusão: doentes seguidos na referida Unidade em programa de OLD contínua ou durante o sono, depois de otimização terapêutica, com duração mínima de três meses. Não foram estabelecidos limites de idade. Excluíram-se doentes com necessidade de oxigenoterapia episódica durante exacerbações infeciosas.

Analisámos variáveis relacionadas com a criança: género, doença de base, antecedentes de prematuridade, comorbilidades (refluxo gastroesofágico, hipertensão pulmonar, apneia obstrutiva do sono) e idade de início e duração de oxigenoterapia. Foram registados os tipos de insuficiência respiratória (IR) (hipoxémica versus global), tipo de administração (contínua versus associada aos períodos de sono), fonte de oxigénio (líquido, gasoso, concentrador), interfaces utilizadas e necessidade de suporte ventilatório domiciliário. Registámos critérios utilizados para prescrição de OLD e monitorização do doente (evolução ponderal e oximetria de pulso). Como marcadores de evolução considerámos adequação ponderal, descontinuação de oxigenoterapia e destino (alta, seguimento, abandono, morte). Analisámos o tempo de acompanhamento dos doentes.

Considerámos os seguintes grupos de diagnóstico, decorrente da experiência internacional ${ }^{3,4,7,8}$ e do conhecimento prévio da casuística da Unidade: ${ }^{5}$ displasia broncopulmonar (DBP), definida pela necessidade de oxigénio num bebé prematuro depois das 36 semanas de idade pós-menstrual, ou seja, com início numa idade cronológica de 0 dias; ${ }^{7}$ bronquiolite obliterante (BO); doença neurológica; fibrose quística $(F Q)$; síndromes polimalformativas; doença de células falciformes (DCF); outras doenças pulmonares neonatais (DP neonatais) e doenças pulmonares do interstício (DPI).

Analisámos os dados através do software estatístico SPSS $^{\circledR}$, versão 19.0 para Windows (Armonk, NY: IBM Corp.) com estatística descritiva e comparativa, nível de significância de $5 \%$. Utilizámos as variáveis skewness e kurtosis para verificar a normalidade das distribuições. $\mathrm{Na}$ comparação entre estudos utilizámos o teste não-paramétrico de Mann-Whitney.

Analisámos orientações clínicas e consensos para oxigenoterapia em idade pediátrica (1 mês - 18 anos), pesquisando na PubMed referências incluídas no período 2003-2013, redigidas em línguas europeias. Considerámos a NOC 018/2011 da DGS ${ }^{11}$ e avaliámos áreas omissas relevantes para a idade pediátrica.

\section{RESULTADOS}

No período 2003-2012 foram acompanhados 86 doentes, $51(59,3 \%)$ rapazes, todos transitados para o domicílio em programa de OLD. Todos foram acompanhados pela Unidade Móvel de Apoio Domiciliário (UMAD).

O principal diagnóstico foi DBP (46; $53,5 \%$ ); seguiu-se BO $(12 ; 14,0 \%)$, maioritariamente pós-infeciosa, apenas num caso associada a reação enxerto versus hospedeiro após transplante medular; doença neurológica (nove; $10,5 \%$ ): quatro doentes com paralisia cerebral (três com encefalopatia hipóxico-isquémica, um com lisencefalia), dois com doença neurometabólica, um com distrofia miotónica de Steinert e DBP, um com atrofia espinhal tipo I e um com síndrome de Rett e epilepsia grave. Dos restantes doentes, sete $(8,1 \%)$ tinham $F Q$; cinco $(5,8 \%)$ síndromes polimalformativas: malformação facial e fenda palatina, mielomeningocelo, malformação de Arnold-Chiari e laringotraqueomalácia, e três cromossomopatias; DCF (três; 3,5\%); outras DP neonatais (dois; $2,3 \%$ ): hipoplasia pulmonar e sequelas de pneumonia grave; e DPI (dois; 2,3\%): hiperplasia das células neuroendócrinas da infância e pneumonite intersticial da infância (Tabela 1).

Para além dos doentes com DBP, sete doentes eram 
Tabela 1 - Caracterização demográfica por diagnóstico da doença de base e mediana da idade no início e duração de oxigenoterapia, no período de 2003-2012

\begin{tabular}{|c|c|c|c|c|}
\hline \multirow{2}{*}{ Diagnósticos } & \multicolumn{2}{|c|}{ Número de doentes } & \multirow{2}{*}{$\begin{array}{l}\text { Idade de início OLD* } \\
\text { (mínimo - máximo) }\end{array}$} & \multirow{2}{*}{$\begin{array}{c}\text { Duração OLD* } \\
\text { (mínimo - máximo) }\end{array}$} \\
\hline & Total (\%) & Género masculino $n(\%)$ & & \\
\hline DBP & $46(53,5)$ & $30(65,2)$ & $0,0(0-22)$ & $11,0(4-51)$ \\
\hline BO & $12(14,0)$ & $9(75,0)$ & $13,5(0-167)$ & $45,0(3-223)$ \\
\hline Doença neurológica & $9(10,5)$ & $4(44,4)$ & $8,0(0-188)$ & $26,0(3-96)$ \\
\hline FQ & $7(8,1)$ & $3(42,9)$ & $168,0(119-216)$ & $24,0(7-43)$ \\
\hline Síndromes polimalformativas & $5(5,8)$ & $2(40,0)$ & $0,0(0-7)$ & $25,0(9-46)$ \\
\hline DCF & $3(3,5)$ & $2(66,7)$ & $108,0(94-136)$ & $15,0(6-20)$ \\
\hline DP neonatais & $2(2,3)$ & $0(0,0)$ & $6,5(0-13)$ & $9,0(5-13)$ \\
\hline DPI & $2(2,3)$ & $1(50,0)$ & $5,0(3-7)$ & $10,5(3-18)$ \\
\hline TOTAL & $86(100,0)$ & $51(59,3)$ & $0,0(0-216)$ & $15,0(3-223)$ \\
\hline
\end{tabular}

* mediana em meses. DBP: displasia bronco-pulmonar; BO: bronquiolite obliterante; FQ: fibrose quística; DCF: doença de células falciformes; DP neonatais: outras doenças pulmonares neonatais; DPI: doenças pulmonares do interstício.

Tabela 2 - Distribuição dos doentes de acordo com o tipo de insuficiência respiratória no período de 2003-2012

\begin{tabular}{lcc} 
& \multicolumn{2}{c}{ Tipo de insuficiência respiratória } \\
\hline DBP & Hipoxémica $n(\%)$ & $2(4,3)$ \\
BO & $44(95,7)$ & $2(16,7)$ \\
Doença neurológica & $10(83,3)$ & $5(55,6)$ \\
FQ & $4(44,4)$ & $5(71,4)$ \\
SP & $2(28,6)$ & $1(20,0)$ \\
DCF & $4(80,0)$ & $0(0,0)$ \\
DP neonatais & $3(100,0)$ & $0(0,0)$ \\
DPI & $2(100,0)$ & $0(0,0)$ \\
TOTAL & $2(100,0)$ & $15(17,4)$
\end{tabular}

DBP: displasia bronco-pulmonar; BO: bronquiolite obliterante; FQ: fibrose quistica; DCF: doença de células falciformes; DP neonatais: outras doenças pulmonares neonatais; DPI: doenças pulmonares do interstício.

prematuros (quatro com BO, um com DP neonatal, um com doença neurometabólica e uma com síndrome polimalformativa). Vinte e um doentes $(25,0 \%)$ tinham registo de comorbilidades: refluxo gastroesofágico $(14 ; 16,2 \%)$, hipertensão pulmonar (quatro; $4,7 \%$ ) e apneia obstrutiva do sono (três; 3,4 \%).

A mediana da idade de início da OLD foi de zero (0-216) meses, pela frequência dos doentes com DBP $(46 ; 53,5 \%)$. A duração mediana de utilização foi de 13,5 (3-199) meses. Estabelecendo uma barreira aos 12 meses, verifica-se que a maioria dos doentes $(65 ; 75,6 \%)$ iniciou OLD no primeiro ano de vida e $72(83,7 \%)$ antes dos 36 meses. Iniciaram mais tardiamente sete doentes com $\mathrm{FQ}$, três doentes com $\mathrm{DCF}$, dois doentes com $\mathrm{BO}$ e dois doentes neurológicos
(Tabela 1)

Predominou a IR hipoxémica ( $71 ; 82,6 \%)$; na IR global $(15 ; 17,4 \%)$ destacam-se os doentes com FQ e doença neurológica (Tabela 2).

A prescrição de OLD contínua prevaleceu ( $74 ; 86,0 \%)$, com $12(14,0 \%)$ doentes a utilizarem apenas nos períodos de sono (Tabela 3).

$\mathrm{O}$ oxigénio líquido isolado foi a principal fonte $(60$; $69,8 \%)$; nove $(10,5 \%)$ crianças utilizaram só oxigénio gasoso e $11(12,8 \%)$, ambos; sete $(8,1 \%)$ crianças utilizaram concentrador. Nas interfaces, destacam-se os óculos nasais $(58 ; 67,4 \%)$, seguidos da sonda nasofaríngea (24; $27,9 \%$ ) (Tabela 3 ).

Doze $(14,0 \%)$ doentes necessitaram de ventilação não 


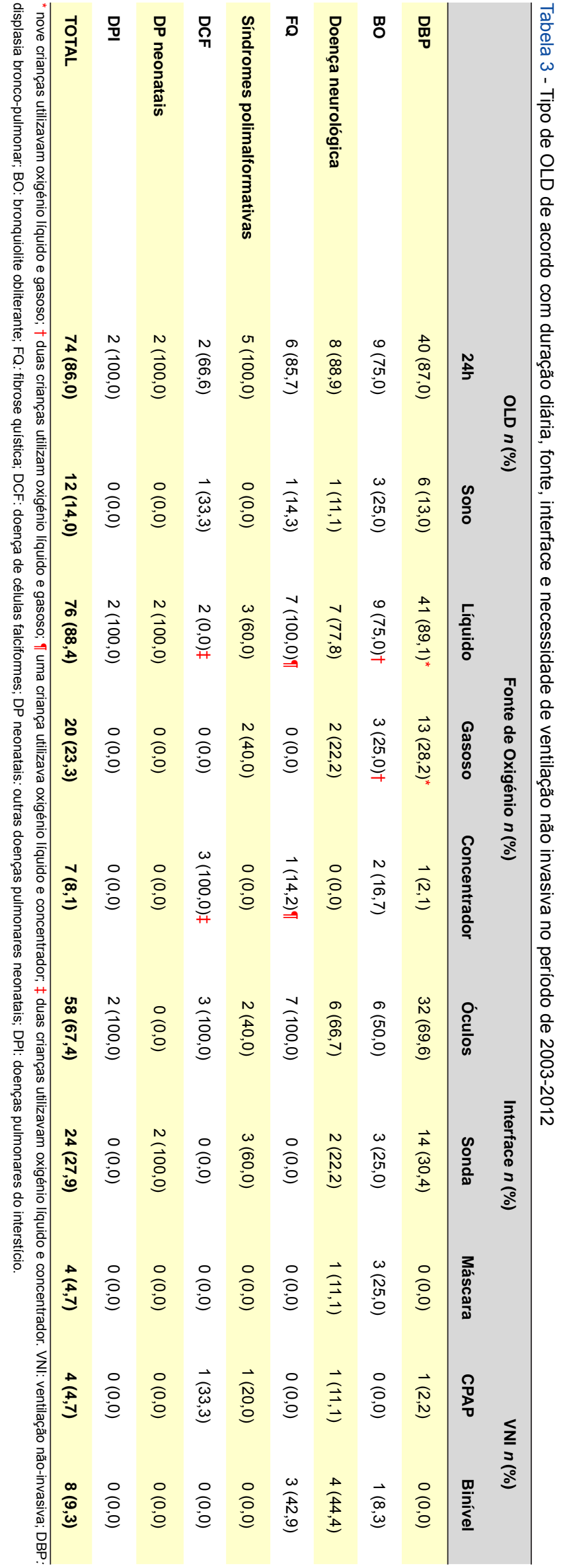

invasiva (VNI) para além da OLD por hipoxémia e/ou hipercapnia persistentes, sobretudo por máscara com dois níveis de pressão (oito; 61,5\%). Os doentes que necessitaram de VNI apresentavam sobretudo doença neurológica (cinco) (Tabela 3). Neste subgrupo foi possível, em nove casos, ter acesso às gasometrias efetuadas previamente, verificando-se $\mathrm{pH}$ mediano de $7,33(7,21-7,34)$ e $\mathrm{pCO}_{2}$ mediano de $69,5 \mathrm{mmHg}(55,2-89,6)$.

Relativamente a critérios para início, monitorização e evolução da OLD, no início do estudo $73(84,9 \%)$ crianças apresentavam peso abaixo do percentil 5 , com 30 crianças desse mesmo grupo a ultrapassar esse limite no final (Tabela 4). Não se encontrou diferença relativamente à evolução ponderal nos diferentes grupos de doença $(p 0,195)$. A oximetria de pulso domiciliária utilizou-se em seis $(6,9 \%)$ doentes. Foi possível descontinuar OLD em $38(44,2 \%)$ (Tabela 4). No final do estudo $12(14,0 \%)$ doentes faleceram, $13(15,1 \%)$ tiveram alta e oito $(9,3 \%)$ abandonaram a consulta (Tabela 4). Faleceram dois doentes com DBP; um com BO associada a febre reumática e hipertensão pulmonar; três doentes neurológicos: atrofia espinhal tipo I, paralisia cerebral com encefalopatia hipóxico-isquémica, síndrome de Rett e epilepsia grave; quatro doentes com FQ e duas síndromes polimalformativas.

O tempo mediano de seguimento foi $39,5(1,0-246,0)$ meses, com a seguinte distribuição crescente por grupos: DP neonatais sete $(1,0-13,0)$ meses, DCF $15,0(6,0-20,0)$ meses, doentes neurológicos 19,0 (4,0-132,0) meses, síndromes polimalformativas $27,0(4,0-40,0)$ meses, DBP $36,5(2,0-246,0)$ meses, DPI 43,0 $(27,0-60,0)$ meses, BO 43,0 (16,0-219,0) meses, FQ 176,0 $(10,5,0-226,0)$ meses. Destaca-se o facto de manterem seguimento após os 18 anos, três doentes com $F Q$, dois doentes neurológicos e um doente com BO.

\section{Comparação de duas décadas de OLD}

Relativamente ao período $1991-2000,{ }^{5}$ destacam-se as seguintes diferenças metodológicas: duração de OLD mínima de três meses para inclusão no estudo (no anterior estabeleceu-se um mês para a DBP); inclusão da doença cardíaca complexa nas síndromes polimalformativas (pela inexistência de doença cardíaca isolada); ausência de representatividade de hipertensão pulmonar primária.

Verifica-se a inclusão de doentes neurológicos e hematológicos e aumento do número de doentes com DBP e BO (Tabela 5). Assiste-se a distribuição semelhante relativamente à idade de início da oxigenoterapia nas diferentes doenças, destacando-se maior duração nos doentes com DBP no período mais recente $(p 0,025)$ (Tabela 6$)$.

\section{Orientações Pediátricas e NOC da DGS}

Foram revistas orientações pediátricas para prescrição de oxigénio domiciliário publicadas em França, ${ }^{3}$ Brasil, ${ }^{4}$ Reino Unido ${ }^{6,8}$ e Espanha. ${ }^{7}$

As diferenças fundamentais para a prescrição pediátrica de OLD referem-se à avaliação, prescrição, monitorização e prognóstico ${ }^{6}$ que, com exceção da FQ e das doenças 
Tabela 4 - Caracterização da evolução dos doentes relativamente à descontinuação de OLD, evolução ponderal e destino, no período de 2003-2012

\begin{tabular}{|c|c|c|c|c|c|c|c|}
\hline & \multicolumn{2}{|c|}{$\begin{array}{l}\text { Evolução ponderal } \\
\qquad n(\%)\end{array}$} & \multirow{2}{*}{$\begin{array}{c}\text { Descontinuação } \\
\text { de OLD } \\
n(\%)\end{array}$} & \multirow[b]{2}{*}{ Seguimento } & \multicolumn{2}{|c|}{$\begin{array}{l}\text { Destino } \\
n(\%)\end{array}$} & \multirow[b]{2}{*}{ Morte } \\
\hline & $\begin{array}{l}\text { Ganho } \\
\text { (> P5) }\end{array}$ & $\begin{array}{l}\text { Perda } \\
\text { (< P5) }\end{array}$ & & & Alta & Abandono & \\
\hline DBP & $15(32,6)$ & $0(0,0)$ & $9(19,6)$ & $27(58,7)$ & $11(23,9)$ & $6(13,0)$ & $2(4,3)$ \\
\hline BO & $6(50,0)$ & $1(8,3)$ & $7(58,3)$ & $9(75,0)$ & $1(8,3)$ & $1(8,3)$ & $1(8,3)$ \\
\hline Doença neurológica & $4(44,4)$ & $0(0,0)$ & $7(77,8)$ & $6(66,7)$ & $0(0,0)$ & $0(0,0)$ & $3(33,3)$ \\
\hline FQ & $0(0,0)$ & $0(0,0)$ & $6(85,7)$ & $3(33,3)$ & $0(0,0)$ & $0(0,0)$ & $4(57,1)$ \\
\hline Síndromes polimalformativas & $1(20,0)$ & $0(0,0)$ & $4(80,0)$ & $3(60,0)$ & $0(0,0)$ & $0(0,0)$ & $2(40,0)$ \\
\hline DCF & $1(33,3)$ & $2(66,7)$ & $3(100,0)$ & $3(0,0)$ & $0(0,0)$ & $0(0,0)$ & $0(0,0)$ \\
\hline DP neonatais & $2(100,0)$ & $2(100,0)$ & $2(100,0)$ & $2(100,0)$ & $0(0,0)$ & $0(0,0)$ & $0(0,0)$ \\
\hline DPI & $1(50,0)$ & $1(50,0)$ & $0(0,0)$ & $0(0,0)$ & $1(50,0)$ & $1(50,0)$ & $0(0,0)$ \\
\hline TOTAL & $30(34,9)$ & $6(7,0)$ & $38(44,2)$ & $53(61,6)$ & $13(15,1)$ & $8(9,3)$ & $12(14,0)$ \\
\hline
\end{tabular}

P5: percentil 5; DBP: displasia bronco-pulmonar; BO: bronquiolite obliterante; FQ: fibrose quística; DCF: doença de células falciformes; DP neonatais: outras doenças pulmonares neonatais; DPI: doenças pulmonares do interstício.

Tabela 5 - Distribuição dos diagnósticos da doença de base dos doentes em OLD, comparando duas décadas (1991-2000 e 2003-2012)

\begin{tabular}{lcc}
\hline & $\begin{array}{c}1991-2001 \\
n(\%)\end{array}$ & $\begin{array}{c}2002-2012 \\
n(\%)\end{array}$ \\
\hline DBP & $40(69,0)$ & $46(53,5)$ \\
FQ & $8(13,8)$ & $7(8,1)$ \\
BO & $4(6,9)$ & $12(14,0)$ \\
DPI & $1(1,7)$ & $2(2,3)$ \\
Doença cardíaca complexa & $1(1,7)$ & -- \\
HTP primária & $1(1,7)$ & $9(10,5)$ \\
Doença neurológica & --- & $5(5,8)$ \\
Síndromes polimalformativas & $3(5,2)$ & $3(3,5)$ \\
DCF & -- & $2(2,3)$ \\
DP neonatais & -- & $\mathbf{8 6}(100,0)$ \\
TOTAL & $\mathbf{5 8 ( 1 0 0 , 0 )}$ & 5
\end{tabular}

DBP: displasia bronco-pulmonar; BO: bronquiolite obliterante; FQ: fibrose quística; DCF: doença de células falciformes; DP neonatais: outras doenças pulmonares neonatais; DPI: doenças pulmonares do interstício.

neuromusculares, é bom em idade pediátrica. Destacam-se, ainda, particularidades pediátricas: 1) possibilidade de descontinuação de oxigenoterapia com o crescimento e necessidade de oxigénio apenas durante o sono (inferior às 15 horas determinadas para o adulto); 2) importância atribuída ao crescimento e desenvolvimento neuro-comportamental, particularmente nas crianças abaixo dos três anos; 3) monitorização preferencial com oximetria de pulso em detrimento da gasometria arterial; 4) prescrição de OLD intermitente se $\mathrm{SpO}_{2}$ abaixo de $90 \%$ durante mais de 5\% do tempo de sono; 5) necessidade de equipamentos para baixos débitos de oxigénio e interfaces apropriadas; 6 ) for- necimento de oxigenoterapia na escola; 7) necessidade de supervisão por um adulto. ${ }^{4}$

A NOC da DGS 018/2011: Cuidados Respiratórios Domiciliários: Prescrição de Oxigenoterapia ${ }^{11}$ descreve algumas destas diferenças e especifica a necessidade de acompanhamento destes doentes em centros especializados; no entanto, a informação é escassa e encontra-se dispersa. Os indicadores de avaliação da implementação da norma não são aplicáveis a toda a idade pediátrica. A inexistência de um fluxograma, bem como a inexistência de diagnósticos específicos de crianças mais novas na folha de prescrição são, também, limitações potenciais. 
Tabela 6 - Distribuição da mediana de idade de início e duração de OLD, de acordo com a doença de base, comparando duas décadas (1991-2000 e 2003-2012)

\begin{tabular}{|c|c|c|c|c|c|c|}
\hline & \multicolumn{3}{|c|}{$\begin{array}{l}\text { Idade de início } \\
\text { (mínimo - máximo) }\end{array}$} & \multicolumn{3}{|c|}{$\begin{array}{c}\text { Duração } \\
\text { (mínimo - máximo) }\end{array}$} \\
\hline & 1991-2000 & 2003-2012 & Valor-p & 1991-2000 & 2003-2012 & Valor-p \\
\hline DBP & $0(0-7)$ & $0(0-22)$ & 0,356 & $9(1-60)$ & $11(4-51)$ & 0,025 \\
\hline FQ & $230(140-297)$ & $168(119-216)$ & 0,118 & $28(10-59)$ & $24(7-43)$ & 0,354 \\
\hline BO & $5(0-25)$ & $13,5(0-167)$ & 0,274 & $15(13-23)$ & $45(3-223)$ & 0,101 \\
\hline DPI & 4 & $5(3-7)$ & 0,439 & 26 & $10,5(1-18)$ & 1,000 \\
\hline Doença cardíaca complexa & 8 & --- & --- & 2 & --- & --- \\
\hline HTP primária & 9 & --- & --- & 2 & --- & --- \\
\hline Doença neurológica & ---- & $8(0-188)$ & --- & --- & $26(3-96)$ & --- \\
\hline Síndromes polimalformativas & $2(1-3)$ & $0(0-7)$ & 0,153 & $15(6-18)$ & $25(9-46)$ & 0,297 \\
\hline DCF & --- & $108(94-136)$ & --- & --- & $15(6-20)$ & --- \\
\hline DP neonatais & --- & $6,5(0-13)$ & --- & --- & $9(5-13)$ & --- \\
\hline TOTAL & $0(0-297)$ & $0(0-216)$ & & $12(1-60)$ & $15(3-223)$ & \\
\hline
\end{tabular}

DBP: displasia bronco-pulmonar; FQ: fibrose quística; BO: bronquiolite obliterante; DPI: doenças pulmonares do interstício; HTP: hipertensão pulmonar; DCF: doença de células falciformes; DP neonatais: outras doenças pulmonares neonatais.

Os limites de prescrição, embora difiram ligeiramente entre as orientações, definem que oxigénio suplementar deve ser prescrito na DBP e outras DP neonatais para $\mathrm{SpO}_{2} \geq 93 \%$; na FQ admite-se $\mathrm{SpO}_{2}>90 \%$ e na DCF $\mathrm{SpO}_{2}$ $>94 \% .{ }^{4,6,7}$ A periodicidade de monitorização difere sobretudo nos lactentes com doenças pulmonares crónicas, com indicação de vigilância e monitorização assíduas, quer no domicílio quer no hospital, para aferição de débitos de oxigénio. . $3,6,7$ Pressupõem-se duas visitas na primeira semana após a alta e intervalos posteriores não superiores a 3-4 semanas. $^{4,6}$

\section{DISCUSSÃO}

Este estudo proveniente de um único centro apresenta uma amostra significativa de doentes pediátricos em OLD com grande variabilidade diagnóstica da condição de base. Verificou-se um ligeiro predomínio do género masculino, tal como referido por outros autores. ${ }^{2}$

A maioria dos doentes iniciou OLD no primeiro ano de vida, acompanhando a maior prevalência de diagnósticos neonatais. Verifica-se, tal como anteriormente, dois picos com grupos etários diferentes no início da OLD. Os diagnósticos associados a OLD precoce na criança associam-se predominantemente a bebés prematuros ou com malformações congénitas; pela sua frequência relativamente ao grupo de entrada em OLD mais tardio, condicionam uma mediana de idades coincidente com zero, sendo este um dos fatores de distinção da OLD em idade pediátrica.

Os doentes que iniciaram OLD após os 36 meses apre- sentavam doenças que, ou ocorreram mais tarde, como a bronquiolite obliterante pós-infecciosa (BO), ou são consequência de progressão da doença, por deterioração da função pulmonar e/ou entrega de oxigénio aos tecidos ( $F Q$, drepanocitose e doença neurológica). A mediana de idade de início e de duração da OLD manteve-se constante ao longo das duas décadas, diferindo apenas em relação à duração na DBP ( $p=0,025)$; esta diferença apresenta um viés de seleção: no estudo prévio considerou-se duração mínima de um mês de OLD, ao passo que no presente foram considerados três meses. Contudo, esta seria uma diferença expectável, considerando a menor idade gestacional do estudo actual, acompanhando a tendência geral da redução do limiar de sobrevivência dos prematuros.

A DBP foi o principal diagnóstico de base para OLD, sendo também o grupo com maior número de doentes a descontinuá-la $(35 ; 80,4 \%)$, tal como observado em Inglaterra e País de Gales. ${ }^{9} \mathrm{O}$ aumento da prematuridade em Portugal, situado nos $9 \%,{ }^{13}$ com estratégias que aumentam a sobrevida de pretermos, ${ }^{14}$ aumentam os casos de DBP. ${ }^{14}$ Propõe-se OLD nestes doentes se $\mathrm{SpO}_{2} \leq 93 \%$, sem hipercapnia excessiva. ${ }^{4,6-8}$ Neste doentes, a OLD é considerada fator determinante na redução da morte súbita no lactente, ${ }^{4,6,7,16}$ da frequência de dessaturações intermitentes, ${ }^{6,7,16}$ da hipertensão pulmonar, ${ }^{6,7}$ e promotor do crescimento ${ }^{7,16-18}$ e neurodesenvolvimento. ${ }^{7,16}$ Nos prematuros, o oxigénio é especialmente tóxico, como demonstrado em estudos prévios (STOP-ROP e BOOST); ${ }^{20,21}$ estabelece-se limite máximo de $\mathrm{SpO}_{2}$ em $95 \%$ para reduzir a incidência de 
retinopatia e da própria $\mathrm{DBP} .{ }^{14} \mathrm{~A}$ duração máxima de oxigenoterapia destes doentes, no presente estudo, foi de 51,0 meses; quando persiste a necessidade após os 12 meses devem ser pesquisadas comorbilidades: laringotraqueomalácia, refluxo gastroesofágico, aspiração recorrente, $F Q$ e doença cardíaca congénita. ${ }^{6} \mathrm{~A}$ mortalidade na DBP foi baixa $(4,3 \%)$, devendo-se sobretudo a comorbilidades decorrentes da prematuridade.

O segundo diagnóstico mais frequente foi $\mathrm{BO}$, diferindo do estudo anterior. ${ }^{5}$ Esta observação acompanha a tendência nacional, ${ }^{22}$ com acompanhamento em unidades especializadas, processo corrente noutros países. ${ }^{2,8}$ Sendo a BO frequentemente adquirida após infeção por adenovírus, ocorre tardiamente. Também aqui a evolução é frequentemente favorável, com cerca de metade dos doentes independentes de OLD no final do estudo.

A doença neurológica foi o terceiro diagnóstico mais frequente. Esta realidade acompanha as crescentes indicações para OLD, equiparando-se ao descrito noutros países da Europa. ${ }^{3,7,9}$ A necessidade de OLD neste doentes decorre da doença pulmonar associada a pneumonias de aspiração, refluxo gastroesofágico, tosse ineficaz e deformidade torácica. ${ }^{4,7}$ Verificámos associação a VNI em cinco doentes, resultado de alterações ventilatórias que condicionam hipoventilação crónica. ${ }^{7}$ Nos doentes neurológicos a OLD tem lugar nos casos ligeiros e não progressivos, na impossibilidade ou ausência de indicação para VNI e nos casos em que, apesar da VNI, o doente mantém hipoxemia. ${ }^{4}$ A mortalidade é elevada $(33,3 \%)$ como referenciado por outros autores.

A melhoria de cuidados respiratórios e nutricionais na área da $F Q$ poderá justificar a redução relativa de OLD em idade pediátrica, pelo atraso verificado no início da insuficiência respiratória. ${ }^{23} \mathrm{Um}$ estudo realizado no $\mathrm{Brasil}^{2}$ revelou a FQ como principal indicação para OLD. Contudo, não está definida a altura ideal para iniciar OLD, não existindo orientações quanto ao rastreio de hipoxemia noturna. ${ }^{24}$ Este grupo ${ }^{4}$ sugere a utilização de critérios para OLD, adultos com doença pulmonar obstrutiva crónica $\left(\mathrm{PaO}_{2} \leq 55 \mathrm{mmHg}\right.$ ou $\mathrm{SpO}_{2} \leq 88 \%$; na presença de cor pulmonale admitir $\mathrm{PaO}_{2}$ 56-59 mmHg ou $\mathrm{SpO}_{2} 89 \%$ ) para adolescentes e dos critérios da $\mathrm{DBP}\left(\mathrm{SpO}_{2} \leq 93 \%\right)$ para lactentes e idade pré-escolar. A frequência de dessaturações não está diretamente relacionada com a gravidade da doença pulmonar, progressão da doença, frequência de hospitalizações ou mortalidade, embora possa influenciar o aproveitamento escolar. $\mathrm{Na}$ nossa série foi possível descontinuar com sucesso a OLD numa doente após transplante pulmonar. Apesar dos avanços terapêuticos e tecnológicos, a FQ continua a ser uma doença grave associada a complicações multiorgânicas, refletindo-se na elevada mortalidade observada no grupo de doentes submetidos a OLD $(57,1 \%)$.

As síndromes polimalformativas apresentam idade de entrada em OLD precoce (0 - 7 meses) e duração prolongada (9 - 46 meses). A complexidade destes casos associa-se também a elevada mortalidade $(40,0 \%)$.

O acompanhamento dos doentes com DCF em centros especializados, já sugerido em orientações internacionais, ${ }^{24}$ e os potenciais benefícios da OLD neste grupo de doentes, justificam a sua inclusão. A prescrição de OLD por hipoxemia intermitente diminui o risco de acidente vascular cerebral, crises dolorosas, hipertensão pulmonar secundária e mortalidade. ${ }^{6}$ De acordo com as linhas orientadoras britânicas, ${ }^{6}$ deve ser avaliada a $\mathrm{SpO}_{2}$ noturna se existir roncopatia ou enurese noturna após os seis anos de idade; é, ainda, recomendada a avaliação anual da $\mathrm{SpO}_{2}$ em crianças estáveis, justificando-se monitorização noturna se $<95 \%$. O mecanismo de dessaturação não é bem compreendido, relacionando-se possivelmente com valores baixos de hemoglobina e consequente desvio para a direita da curva de dissociação da hemoglobina, ou com presença de carboxi e metahemoglobinas ${ }^{25,26}$ A medição da $\mathrm{SpO}_{2}$ pode estar sobrestimada e não ser fiável, ${ }^{26,27}$ recomendando-se que a $\mathrm{SpO}_{2}$ seja aferida durante o período de sono e vigília; se $<93 \%$ deve aferir-se através da $\mathrm{PaO}_{2}$ : se $<70 \mathrm{mmHg}$ a OLD deve ser considerada. ${ }^{4} \mathrm{~A}$ necessidade de OLD surge com a evolução da doença, pelo que a idade de início é tardia, não tendo sido possível descontinuar OLD em nenhum doente durante o período do estudo. Uma das crianças apresentava concomitantemente apneia obstrutiva do sono, necessitando de VNI até à adenoamigdalectomia.

Como outras DP neonatais foram incluídas uma hipoplasia pulmonar e sequelas de pneumonia grave. São utilizados os mesmos critérios para início de oxigenoterapia adotados para crianças com DBP, uma vez que atinge predominantemente lactentes. ${ }^{6}$ Ambas as situações apresentam pouco tempo de seguimento, mantendo necessidade de OLD à data de colheita dos dados (duração de 5 e 13 meses). Embora nenhuma das crianças tenha ficado independente de OLD, estudos demonstram que a suspensão de oxigénio é frequente após 12 a 28 meses (14 - 66 meses). ${ }^{28,29}$

As DPI são representadas neste estudo por um caso de hiperplasia congénita das células neuroendócrinas da infância e um de pneumonia intersticial inespecífica. O compromisso das trocas gasosas obriga à suplementação com oxigénio durante períodos variáveis, enquanto é realizada terapêutica dirigida.

A utilização dos dispositivos de oxigénio, a monitorização e a aferição do débito de oxigénio suplementar no doente pediátrico em OLD apresenta algumas diferenças relativamente ao doente adulto que se especificam de seguida.

Verificámos predomínio do oxigénio líquido. Estes dispositivos portáteis permitem a deambulação e são fáceis de recarregar no domicílio. ${ }^{4}$ A principal desvantagem é o elevado custo. ${ }^{4,6,7} A$ alternativa mais económica consiste no oxigénio gasoso em cilindros, embora menos prático: volumoso e necessidade de recargas frequentes, dificultando a autonomia e deambulação, fundamental na maior parte destas crianças que têm um futuro com funcionalidade normal. ${ }^{7}$ Outras alternativas são o concentrador, ${ }^{4}$ pouco utilizado em crianças: permitem pouca mobilidade, necessita de energia elétrica e são ruidosos. ${ }^{7}$ 
Os sistemas de administração / interfaces variam entre óculos nasais, sonda nasofaríngea e máscara facial. Neste estudo os sistemas mais utilizados foram as cânulas nasais (óculos nasais e sonda nasofaríngea), tal como descrito por outros autores. ${ }^{2}$ As cânulas nasais são o método preferencial pela sua comodidade. Devem ser mudadas frequentemente ${ }^{4}$ com limpeza diária. As cânulas nasais evitam o desperdício de oxigénio e facilitam a comunicação e ingestão de comida. ${ }^{6,7}$ Para débitos $>2$ litros/minuto as cânulas tornam-se desconfortáveis, estando indicada a máscara facial ${ }^{4,6,7}$ A escolha depende da doença respiratória e da necessidade de oxigénio, podendo incluir a preferência dos pais. ${ }^{6}$

A monitorização do doente pediátrico em OLD inclui a pesquisa e avaliação frequente das consequências da hipoxemia crónica e é diferente na criança relativamente ao adulto ${ }^{3,6,7}$ uma vez que a repercussão mais relevante é alteração do crescimento estaturo-ponderal e do desenvolvimento cognitivo, para além dos habituais aumentos do hematócrito, hipertensão arterial pulmonar e hipertrofia do ventrículo direito. Nas normas orientadoras francesas ${ }^{3}$ é sugerida avaliação do doente crónico durante o período de sono (6 - 12h) e de atividades; também o valor de $\mathrm{CO}_{2}$ diurno deve ser monitorizado e, se normal, de $\mathrm{CO}_{2}$ noturno. Em crianças abaixo dos dois anos consideram indicação para OLD a presença de $\mathrm{SpO}_{2}<93 \%$ e/ou mais de $5 \%$ do período de sono abaixo de $90 \%$ e/ou presença de hipertensão pulmonar arterial. Em crianças acima dos dois anos sugerem $\mathrm{SpO}_{2}<90 \%$ ou mais de $10 \%$ do período de sono abaixo de $90 \%$ e/ou hipertensão pulmonar arterial; e valores alvo para OLD a $\mathrm{SpO}_{2}>94 \%$ ou $>92 \%$ consoante exista ou não hipertensão pulmonar arterial.

Neste estudo, a monitorização dos doentes consistiu na oximetria de pulso em regime de hospital de dia ou visita domiciliária e na evolução antropométrica. As gasometrias sanguíneas representaram um escasso número, sublinhando que, ao contrário dos adultos em OLD, a gasometria sanguínea não deve ser utilizada como critério para prescrição em idade pediátrica. Deve conhecer-se o tipo de oxímetro ${ }^{6}$ utilizado (saturação fraccional ou funcional) ${ }^{6}$ e as suas limitações. ${ }^{4}$ A utilização de oxímetro de pulso em ambulatório não traz benefícios para o doente pediátrico em OLD, ${ }^{6}$ tendo sido alvo de revisão anterior. ${ }^{30}$

Relativamente à evolução antropométrica, 30 (34,9\%) crianças ultrapassaram o percentil 5 no decurso da OLD, evidenciando a sua importância e benefício global.

O doente pediátrico em OLD, pelas suas especificidades, deve ter o apoio de uma equipa domiciliária. Embora transcendendo o objetivo do presente estudo, de referir que os doentes em OLD acompanhados na Unidade têm visita domiciliária da UMAD. Esta resulta de uma parceria institucional entre a Fundação do Gil e o Hospital de Santa Maria, já no nono ano de existência, que permite a visita periódica de um enfermeiro hospitalar. Uma vez que a quantidade de doentes pediátricos com cuidados técnicos respiratórios é menor quando comparado com o grupo de adultos, e dada a grande diversidade da doença de base, acredita-se no benefício da visita domiciliária proveniente da instituição hospitalar de acompanhamento. Nas visitas de monitorização destes doentes efectua-se a avaliação da $\mathrm{SpO}_{2}$ em ar ambiente e com oxigénio. ${ }^{4}$ Num estudo ${ }^{31}$ onde foram incluídas 55 crianças com DBP e avaliados os cuidados domiciliários, $41 \%$ das crianças necessitaram de um reinternamento, com mediana de internamento de nove dias.

A descontinuação de OLD deve equacionar-se quando o suplemento de oxigénio é $<0,1 \mathrm{~L} /$ minuto, utilizando-se as saturações alvo que conduziram à sua instituição. ${ }^{4,6,7}$ É regra na DBP, e também em casos de DPI e BO.,4, No presente estudo a OLD foi descontinuada em 38 (44,2\%) doentes. Dependendo da situação, a descontinuação de OLD pode passar inicialmente por um regime noturno ou logo nas $24 \mathrm{~h}$, não existindo consenso relativamente à meIhor estratégia., ${ }^{4,6,8}$ Os equipamentos devem permanecer no domicílio pelo menos três meses após descontinuação. Após esse período, deve ser realizada uma avaliação com oximetria de pulso em duas ocasiões com cerca de um mês de intervalo e da evolução ponderal da criança. ${ }^{4}$

Não podemos deixar de referir o seguimento de doentes para além dos 18 anos, nomeadamente doentes com $\mathrm{FQ}$, doença neurológica e $\mathrm{BO}$, facto que se prende com as particularidades e dificuldades existentes na transição de doentes crónicos para a medicina de adultos. Esta realidade é melhor enfrentada quando existe um tempo de sobreposição de seguimentos, de acompanhamento paralelo, permitindo ao doente e à família a adaptação progressiva à nova realidade. Por esse motivo, incluímos doentes que ultrapassam a idade pediátrica, mas que continuam a manter seguimento na Unidade estudada.

Por ser um estudo retrospetivo e de longa duração, uma das suas limitações é a precisão dos registos. Possivelmente nem todos os doentes seguiram orientações sobreponíveis, nem periodicidades de observação constantes. A existência de um sistema estruturado de prescrição, imprescindível para o planeamento desta terapêutica, ultrapassaria esta limitação. Relativamente à comparação das duas décadas, os grupos nosológicos diferentes impedem uma comparação direta. Por outro lado, os critérios de inclusão relativamente à DBP foram diferentes, pelo que estamos perante um viés de seleção.

\section{CONCLUSÕES}

Acompanhando os relatos internacionais e descrições anteriores, os doentes pediátricos com maior benefício com programas de OLD são portadores de DBP, com evolução favorável e possibilidade de descontinuação. A melhoria dos cuidados ao doente com FQ traduz-se na redução destes doentes sob OLD em idade pediátrica. A inclusão de novos grupos de diagnóstico pode traduzir a centralização no acompanhamento.

As especificidades na utilização da OLD em crianças relativamente aos adultos são diversas, e exigem um maior consumo de recursos, pela dinâmica de crescimento e pela diversidade de doenças subjacentes à OLD. 
A criação de um sistema consistente e homogéneo de prescrição de OLD permitiria a elaboração de uma base nacional de registos, com caracterização e conhecimento da população pediátrica em OLD; este processo criaria homogeneidade nos critérios e sistemas de prescrição.

Os autores sugerem a criação de orientações específicas para a pediatria e a evolução para registos nacionais rigorosos que permitam o planeamento destes cuidados.

\section{OBSERVAÇÕES}

Os dados do presente trabalho foram parcialmente apresentados nos seguintes congressos:

a) $10^{\text {th }}$ International Congress on Pediatric Pneumology, 25-27/06/2011, Versailles, França. Poster: Long-term

\section{REFERÊNCIAS}

1. Tin W. Oxygen therapy: 50 years of uncertainty. Pediatrics. 2002;110:615-6.

2. Munhoz AS, Adde FV, Nakaie CM, Doria Filho U, Silva Filho LV, Rodrigues JC. Long-term home oxygen therapy in children and adolescents: analysis of clinical use and costs of a home care program. J Pediatr. 2011;87:13-8.

3. Aubertin G, Marguet C, Delacourt C, Houdouin V, Leclainche L, Lubrano $M$, et al. Recommandations pour l'oxygénothérapie chez l'enfant en situations aigues et chroniques : évaluation du besoin, critéres de mise en route, modalités de prescription et de surveillance. Arch Pediatr. 2012;19:528-36.

4. Adde FV, Alvarez AE, Barbisan BN, Guimarães BR. Recommendations for long-term home oxygen therapy in children and adolescents. J Pediatr, 2013;89:6-17.

5. Ferreira R, Bandeira T. Oxigenoterapia de longa duração em pediatria: lições do passado e orientações para o futuro. Acta Pediatr Port. 2003;34:69-78.

6. Balfour-Lynn IM, Field DJ, Gringras P, Hicks B, Jardine E, Jones RC, et al. BTS guidelines for home oxygen in children. Thorax. 2009;64:1-26.

7. Paredes MC, Cruz OA, Aznar IC, Ruiz EP, Frías JP. Fundamentos de la oxigenoterapia en situaciones agudas e crónicas: indicaciones, métodos, controles y seguimiento. An Pediatr. 2009;71:161-74.

8. Balfour-Lynn IM, Field DJ, Gringras P, Hicks B, Jardine E, Jones RC, et al. Home oxygen for children: who, how and when? Thorax. 2005;60:7681.

9. Primhak RA, Hicks B, Shaw NJ, Donaldson GC, Balfour-Lynn IM. Use of home oxygen for children in England and Wales. Arch Dis Child 2011;96:389-92.

10. Greenough A, Alexander J, Burgess S, Chetcuti PA, Cox S, Lenney W, et al. High versus restricted use of home oxygen therapy, health care utilization and the cost of care in chronic lung disease. Eur $\mathrm{J}$ Pediatr. 2004;163:292-6.

11. Norma de Orientação Clínica. Direção Geral de Saúde. Cuidados respiratórios domiciliários: prescrição de oxigenoterapia. Norma $n^{\circ}$ 18/2011 de 28/09/2011 atualizada a 12/02/2013 [consultada $2013 \mathrm{Abr}$ 24]. Disponível em: http://www.dgs.pt/directrizes-da-dgs/normas-ecirculares-normativas/norma-n-0182011-de-28092011-atualizada-a12022013-jpg.aspx.

12. Harrison G, Show B. Prescribing home oxygen. Arch Dis Child Fetal Neonatal. 2007;92:241-3.

13. Machado MC, Alves MI, Couceiro ML. Saúde infantil e juvenil em Portugal: indicadores do Plano Nacional de Saúde. Acta Pediatr Port. 2011;42:195-204.

14. Sweet DG, Carnielli V, Greisen G, Hallman M, Ozek E, Plavka R, et al. European consensus guidelines on the management of neonatal respiratory distress syndrome in preterm infants. Neonatology. 2010;97:402-17.

15. Gupta S, Prasanth K, Chen CM, Yeh TF. Postnatal corticosteroids for oxygen therapy: Review of requirements in children.

b) $12^{\circ}$ Congresso Nacional de Pediatria, 6-8/10/2011, Albufeira, Portugal. Comunicação Oral: Oxigenoterapia de longa duração em idade pediátrica: análise da evolução da utilização clínica.

\section{CONFLITOS DE INTERESSE}

Os autores declararam a inexistência de conflitos de interesse.

\section{FONTES DE FINANCIAMENTO}

O presente trabalho não foi objecto de qualquer bolsa ou subsidio.

prevention and treatment of chronic lung disease in preterm newborn. Int J Pediatr. 2012;315642 [consultado 2013 Abr 12]. Disponível em: http://www.hindawi.com/journals/ijpedi/2012/315642/.

16. Poets CF. When infants need additional inspired oxygen? A review of the current literature. Pediatr Pulmonol. 1998;26:424-8.

17. Kotecha S, Allen J. Oxygen therapy for infants with chronic lung disease. Arch Dis Child Fetal Neonatal. 2002;87:11-4.

18. Groothuis JR, Rosenberg AA. Home oxygen promotes weight gain in infants with bronchopulmonary dysplasia. Am J Dis Child 1987;141:9925.

19. Hudak BB, Allen MC, Hudak ML, Loughlin GM. Home oxygen therapy for chronic lung disease in extremely low-birth weight infants. Am J Dis Child. 1989;143:357-60.

20. Supplemental Therapeutic Oxygen for Prethreshold Retinopathy of Prematury (STOP-ROP), a randomized, controlled trial. I: primary outcomes. Pediatrics. 2000;105:295-310.

21. Askie LM, Henderson-Smart SJ, Irwig L, Simpson JM. Oxygensaturations targets and outcomes in extremely preterm infants. $\mathrm{N}$ Eng J Med. 2003;349:959-67.

22. Bandeira T, Nunes T. Long-term follow-up of chronic lung disease of infancy. Pediatr Pulmonol. 2011;46:573-80.

23. Urquhart DS, Montgomery H, Jaffe A. Assessment of hypoxia in children with cystic fibrosis. Arch Dis Child. 2005;90:1138-43.

24. NHS Sickle cell and thalassemia screening program in partnership with the Sickle Cell Society. Sickle cell disease in childhood. Detailed guidance standards and guidelines for clinical care 2010 [Consultado em 2013 Abr 17]. Disponível em: http://sct.screening.nhs.uk/ standardsandguidelines.

25. Blaisdell CJ, Goodman S, Clark K, Casella JF, Loughlin GM. Pulse oximetry is a poor predictor of hypoxemia in stable children with sickle cell disease. Arch Pediatr Adolesc Med. 2000;154:900-3.

26. Moreira GA. Respiratory repercussions of sickle cell anemia. J Bras Pneumol. 2007;33:18-20.

27. Pianosi P, Charge TD, Esseltine DW, Coates AL. Pulse oximetry in sickle cell disease. Arch Dis Child. 1993;68:735-8.

28. Castro-Rodriguez JA, Daszenies C, Garcia M, Meyer R, Gonzales R. Adenovirus pneumonia in infants and factors for developing bronchiolitis obliterans: a 5-year follow-up. Pediatr Pulmonol. 2006;41:947-53.

29. Norzila MZ, Azizi BH, Norrashidah AW, Yeoh NM, Deng CT. Home oxygen therapy for children with chronic lung diseases. Med J Malaysia. 2001;56:151-7.

30. Saianda A, Estevão MH, Bandeira T. Utilização domiciliária de monitores cardio-respiratórios e oxímetros de pulso em Pediatria. Resultados do inquérito nacional "Monitores cardio-respiratórios em Pediatria". Acta Pediatr Port. 2007;38:73-8.

31. Hallam L, Rudbeck B, Bradley M. Resource use and costs of caring for oxygen-dependent children: a comparison of hospital and home-based care. J Neonatal Nurs. 1996;2:25-30. 


\section{Oxigenoterapia Domiciliária de Longa Duração na Criança: Evidências e Questões em Aberto}

Acta Med Port 2014:27:717-725

Publicado pela Acta Médica Portuguesa, a Revista Científica da Ordem dos Médicos

Av. Almirante Gago Coutinho, 151

1749-084 Lisboa, Portugal.

Tel: +351 218428215

E-mail: submissao@actamedicaportuguesa.com

www.actamedicaportuguesa.com

ISSN:0870-399X | e-ISSN: 1646-0758

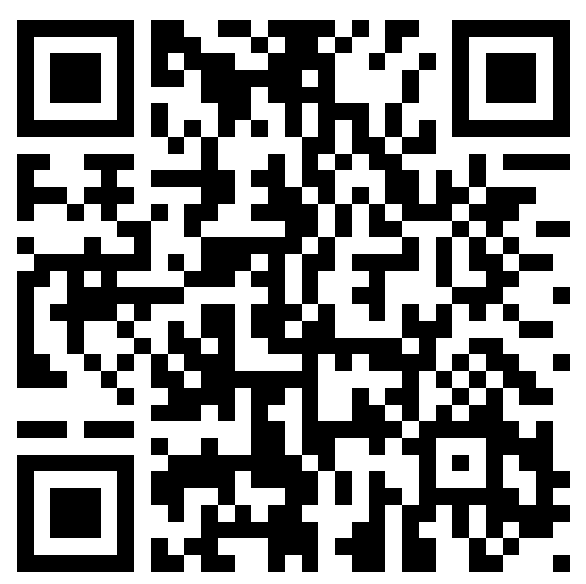

